\title{
Utilizing the Maximum Workload Range for Practice Periodization
}

Commentary

Gabriel J. Sanders ${ }^{1}$, Corey A. Peacock ${ }^{2}$

${ }^{\prime}$ Northern Kentucky University, Highland Heights, Kentucky/USA

${ }^{2}$ Nova Southeastern University, Fort Lauderdale, Florida/USA

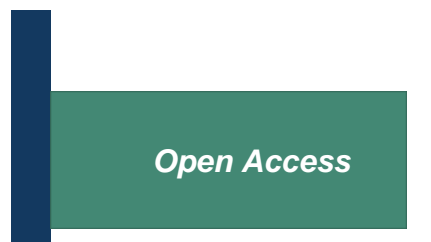

Published: January 20, 2022

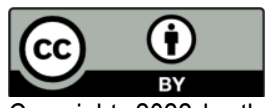

Copyright, 2022 by the authors. Published by Pinnacle Science and the work is licensed under the Creative Commons Attribution 4.0 International License. To view a copy of this license, visit http://creativecommons .org/licenses/by/4.0/

Research Directs in Strength and Performance: 2022, Volume 2 (Issue 1): 1

ISSN: 2768-5187
Key Words: Training loads, overtraining, undertraining

Corresponding author: Gabriel J. Sanders, sandersg1@nku.edu

\section{Commentary}

The maximum workload range (max range) is a concept suggested by Sanders et al. ${ }^{1}$ regarding a method used to prescribe adequate practice workloads based off wearable technology data. The max range is calculated as follows from game data:

Max Range $=($ Mean Total Distance +1 St. Dev. $)$ to (Maximum Total Distance $)$

While the example provided utilizes total distance, the max range can be applied to key performance indicators such as high-speed distance, training load, jumps, etc. that are tracked throughout the competitive season in team sports. The max range concept was developed from research that found $12-17 \%$ of the time, football athletes, depending on position, accumulated game workloads outside their position's mean $+1 \mathrm{SD}{ }^{1}$. Anecdotally, many coaches and practitioners use simple game averages as a control for ideal practice volumes. Based on previous research, using the game average as control training threshold may result in some high performing athletes being under-conditioned ${ }^{1-3}$. It is reasonable to suggest that potential compound effects may occur throughout an entire season if athletes are not engaging in rigorous training loads that mimic game-like volumes and intensities.

Figure 1. Theoretical football periodization structure for a defensive back using the max range for high intensity training days.

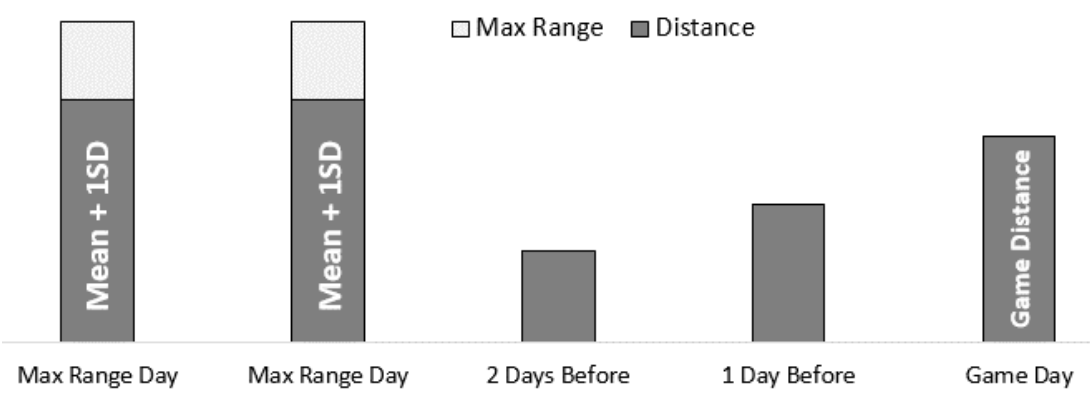

While optimal conditioning is the goal, it should be noted that repetitively training at greater than game-like intensities in any given week may have deleterious effects that lead to fatigue and performance decrements. A case study on an NCAA Division I volleyball athlete found that in losing performances, excessive high intensity jumps in practices 
leading up to a game resulted in a 2 -fold decreases in hitting percentaget. The excessively high jump loads in practices before losing performances in this case were 2-3 times greater than game jump loads 4 .

Furthermore, adjusting intensity and duration workloads for practice is challenging, but determining workloads for optimal performance is valuable for programming and periodization models throughout a season. For example, in basketball, an ideal practice session aimed to mimic game-like intensity is $>85 \%$ of the athlete's maximum heart rate $\left(>85 \% \mathrm{HR}_{\max }\right)^{2,5,6}$. While the intensity threshold (i.e., $>85 \% \mathrm{HR}_{\max }$ ) of basketball training has been identified in several studies ${ }^{2,6}$, the ideal duration of practicing at that intensity for optimal conditioning and performance is relatively understudied and further assessments are warranted.

\section{Conclusion}

In depth analysis of wearable data is emerging and monitoring workloads with wearable technology is being utilized at the highest levels of competitive sports. More research is needed to assess if practicing in the max range 3-4 days prior to competition can potentially impact game performance. While in game performance is multifaceted and varies for every athlete, research should aim to assess controllable factors such as practice intensity and duration ${ }^{7-9}$. Future research should also aim to assess if engaging in the max range, at least one time per week, can reduce the likelihood of injury, as athletes may be more optimally prepared for the rigors and demands of competition.

\section{Acknowledgements}

Thank you to Coach Brad Roll at the University of Tennessee, and Coaches Brian Boos, Frank Shipley and Sarah Crosby at Northern Kentucky University for the collaboration and their work collecting wearable data throughout the years.

\section{References}

1. Sanders GJ, Roll B, Peacock CA, Kollock RO. Maximum Movement Workloads and High-Intensity Workload Demands by Position in NCAA Division I Collegiate Football. J Strength Cond Res. 2020;34(7):19741981.

2. Sanders GJ, Boos B, Rhodes J, Kollock RO, Peacock CA. Competition-Based Heart Rate, Training Load, and Time Played Above 85\% Peak Heart Rate in NCAA Division I Women's Basketball. J Strength Cond Res. 2021;35(4):1095-1102.

3. Sanders GJ, Roll B, Peacock CA. Maximum Distance and High-Speed Distance Demands by Position in NCAA Division I Collegiate Football Games. J Strength Cond Res. 2017;31(10):2728-2733.

4. Sanders GJ, Boos B, Shipley F, Scheadler CM, Peacock CA. An Accelerometer-Based Training Load Analysis to Assess Volleyball Performance. J Exerc Nutri. 2018;1(1).

5. Sanders GJ, Boos B, Rhodes J, Kollock RO, Peacock CA, Scheadler CM. Factors associated with minimal changes in countermovement jump performance throughout a competitive division I collegiate basketball season. J Sports Sci. 2019;37(19):2236-2242.

6. Stojanovic E, Stojiljkovic N, Scanlan AT, Dalbo VJ, Berkelmans DM, Milanovic Z. The Activity Demands and Physiological Responses Encountered During Basketball Match-Play: A Systematic Review. Sports Med. 2018;48(1):111-135.

7. Ben Abdelkrim N, Castagna C, El Fazaa S, El Ati J. The effect of players' standard and tactical strategy on game demands in men's basketball. J Strength Cond Res. 2010;24(10):2652-2662.

8. Ben Abdelkrim N, El Fazaa S, El Ati J. Time-motion analysis and physiological data of elite under-19-yearold basketball players during competition. BrJ Sports Med. 2007;41(2):69-75.

9. Berkelmans DM, Dalbo VJ, Kean CO, et al. Heart Rate Monitoring in Basketball: Applications, Player Responses, and Practical Recommendations. J Strength Cond Res. 2018;32(8):2383-2399. 